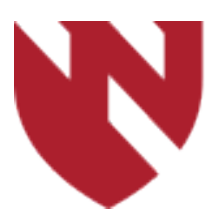

September 2020

\title{
The Diagnostic Utility of a Myeloid Mutational Panel for Myelodysplastic Syndromes and Myelodysplastic/ Myeloproliferative Neoplasms
}

\author{
Warda Ibrar \\ University of Nebraska Medical Center \\ Jesse Cox \\ University of Nebraska Medical Center \\ Catalina Amador \\ University of Nebraska Medical Center \\ Hina N. Qureishi \\ University of Nebraska Medical Center \\ Kai Fu \\ University of Nebraska Medical Center
}

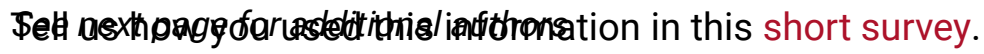

Follow this and additional works at: https://digitalcommons.unmc.edu/gmerj

Part of the Higher Education Commons, and the Medicine and Health Sciences Commons

\section{Recommended Citation}

Ibrar, W., Cox, J., Amador, C., Qureishi, H. N., Fu, K., Greiner, T. C., Vokoun, A., Zhang, W., , Ji, Y. The Diagnostic Utility of a Myeloid Mutational Panel for Myelodysplastic Syndromes and Myelodysplastic/ Myeloproliferative Neoplasms. Graduate Medical Education Research Journal. 2020 Sep 29; 2(1). https://digitalcommons.unmc.edu/gmerj/vol2/iss1/57 


\section{The Diagnostic Utility of a Myeloid Mutational Panel for Myelodysplastic Syndromes and Myelodysplastic/Myeloproliferative Neoplasms}

\section{Creative Commons License}

\section{(c) (i) $\Theta($}

This work is licensed under a Creative Commons Attribution-Noncommercial-No Derivative Works 4.0 License.

\section{Authors}

Warda Ibrar, Jesse Cox, Catalina Amador, Hina N. Qureishi, Kai Fu, Timothy C. Greiner, Allison Vokoun, Weiwei Zhang, and Yuan Ji 
Conclusion: There is a significant association between upper and lower extremity fractures with snow/ice accumulation, but not rain/ melted snow.

\section{The Diagnostic Utility of a Myeloid Mutational Panel for Myelodysplastic Syndromes and Myelodysplastic/ Myeloproliferative Neoplasms \\ Warda Ibrar'1, Jesse Cox¹, Catalina Amador ${ }^{1}$, Hina N. Qureishi', Kai Fu', Timothy C. Greiner ${ }^{1}$, Allison Vokoun', Weiwei Zhang', Yuan Ji ${ }^{1}$ University of Nebraska Medical Center, Department of Pathology}

Mentor: Yuan Ji

Program: Pathology, Division of Hematopathology

Type: Original Research

Background: The diagnosis of myelodysplastic syndromes (MDS) and myelodysplastic/myeloproliferative neoplasms (MDS/MPN) is based on morphology and cytogenetics/FISH findings per 2017 WHO classification. With rare exceptions (e.g. SF3B1 mutations), somatic mutations have not been incorporated as diagnostic criteria. In this study we analyzed the utility of mutational analysis with a targeted 54-gene or 40-gene next generation sequencing (NGS) panel in diagnosis of MDS and MDS/MPN.

Methods: The clinicopathologic data and NGS results of patients with unexplained cytopenia with or without cytosis who underwent a bone marrow (BM) biopsy and had sequencing with either a 54 gene Trusight Myeloid or a 40 gene Oncomine Myeloid NGS Assay performed at our institution from 2017 to 2019. Morphologic diagnosis of BM biopsy based on 2017 WHO classification was considered gold standard.

Results: A total 74 patients were identified, including 25 low-grade MDS (peripheral blood blasts $<1 \%$ and BM blasts $<5 \%$ ), 16 high-grade MDS (peripheral blood blasts 2-19\% and BM blasts 5-19\%), 5 therapyrelated MDS, 14 MDS/MPN, and 14 morphological negative ones. Of 74 patients, 158 somatic mutations involving 37 genes were detected and had variant allele frequency (VAF) ranging from $3 \%$ to $99 \%$. $62 \%$ (23/37) genes showed recurrent mutations and $38 \%(14 / 37)$ genes had one mutation each. The most common mutated genes were TET2, ASXL1, RUNX1, SF3B1 and TP53. Morphological negative, low-grade MDS, high-grade MDS, therapy-related MDS and MDS/MPN showed an average number of somatic mutations with a mean VAF: $1.5 / 20 \%$, $2 / 37 \%, 3.1 / 38 \%, 2 / 33 \%$ and $4.8 / 41 \%$, respectively. Mutations in TP53, RUNX1, or ASXL1 were associated with high-grade and therapy-related MDS $(p=0.004)$, while SF3B1 mutations were associated with low-grade
MDS $(p=0.001)$. In 60 of 74 patients with a diagnosis of MDS or MDS/MPN, 32 showed abnormal cytogenetics and 28 showed normal cytogenetics. One or more mutations were detected in 25 of 28 (89\%) MDS or MDS/ MPN patients with normal cytogenetics. The sensitivity, specificity, and positive predictive value (PPV) and negative predictive value (NPV) of mutations for MDS and MDS/ MPN with different cutoffs are summarized in Table 1.

Conclusion: A myeloid mutational panel provides additional evidence of clonality besides cytogenetics/FISH studies in diagnosis of cytopenia with or without cytosis, and $\geq 2$ mutations with $\geq 10 \%$ VAF highly predicts MDS and MDS/MPN with a PPV of $100 \%$.

https://doi.org/10.32873/unmc.dc.gmerj.2.1.055

\section{Table 1.}

Diagnostic performance of mutations for MDS and MDS/MPN with different cutoffs.

\begin{tabular}{lccccc} 
& $\begin{array}{c}\text { Any mutations } \\
(\mathrm{VAF} \geq 1 \%)\end{array}$ & $\mathrm{VAF} \geq 20 \%$ & $\geq 2$ mutations & $\begin{array}{c}\mathrm{VAF} \geq 10 \% \text { and } \\
\geq 2 \text { mutations }\end{array}$ & $\begin{array}{c}\mathrm{VAF} \geq 10 \% \text { and } \geq 2 \\
\text { mutations, or abnormal } \\
\text { cytogenetics/FISH }\end{array}$ \\
\hline Sensitivity & $\mathbf{8 7 \%}$ & $\mathbf{7 5 \%}$ & $\mathbf{5 8 \%}$ & $\mathbf{5 8 \%}$ & $\mathbf{8 3 \%}$ \\
\hline Specificity & $\mathbf{5 0 \%}$ & $\mathbf{9 3} \%$ & $\mathbf{9 3} \%$ & $\mathbf{1 0 0 \%}$ & $\mathbf{8 6 \%}$ \\
\hline PPV & $\mathbf{8 8 \%}$ & $\mathbf{9 8 \%}$ & $\mathbf{9 8 \%}$ & $\mathbf{1 0 0 \%}$ & $\mathbf{9 6 \%}$ \\
\hline NPV & $\mathbf{4 7 \%}$ & $\mathbf{4 6 \%}$ & $\mathbf{3 4 \%}$ & $\mathbf{3 6 \%}$ & $\mathbf{5 4 \%}$ \\
\hline
\end{tabular}

\section{Coronary Artery Bypass Grafting Versus Percutaneous Coronary Intervention for Left Main Disease in Chronic Kidney Disease Patients}

Aravdeep Jhand ${ }^{1}$, Raahat Bansal2 ${ }^{2}$, Rahul Dhwan ${ }^{1}$, J. Dawn Brown ${ }^{3}$, Thomas Porter ${ }^{1}$, James Tcheng ${ }^{4}$, Yiannis Chatzizsis ${ }^{1}$, Andrew Goldsweig ${ }^{1}$

${ }^{1}$ University of Nebraska Medical Center, Department of Internal Medicine, Division of Cardiovascular Disease

${ }^{2}$ Creighton University, Department of Internal Medicine

${ }^{3}$ Brown University, Department of Internal Medicine, Division of Cardiovascular Disease

${ }^{4}$ Duke University, Department of Internal Medicine, Division of Cardiovascular Disease

Mentor: Andrew Goldsweig

Program: Internal Medicine, Division of Cardiovascular Disease

Type: Review/Meta-analysis

Background: Limited data exists on the optimal revascularization strategy for patients with left main coronary artery disease
(LMCAD) and chronic kidney disease (CKD) We conducted this updated meta-analysis to compare the outcomes of coronary artery bypass graft $(\mathrm{CABG})$ versus percutaneous coronary intervention $(\mathrm{PCI})$ in this population.

Methods: Multiple electronic databases were searched for studies comparing CABG and $\mathrm{PCI}$ in $\mathrm{CKD}$ patients undergoing revascularization of LMCAD $(>50 \%$ diameter stenosis). CKD was defined as estimated glomerular filtration rate of $<60$ $\mathrm{ml} / \mathrm{min} / 1.73 \mathrm{~m} 2$. Primary outcome of interest was long term major adverse cardiovascular and cerebral events (MACCE) which was a composite of cardiovascular death, myocardial infarction (MI), stroke and repeat revascularization. Other outcomes of interest 